\title{
An evidence-based perspective on Lower Urinary Tract Symptoms and telemedicine during the COVID-19 pandemic
}

\author{
Linda Collins $^{1}\left(\mathbb{D} \cdot\right.$ Rajvinder Khasriya $^{2} \cdot$ James Malone-Lee $^{2}$
}

Received: 14 April 2021 / Accepted: 4 July 2021 / Published online: 17 July 2021

(c) The Author(s) 2021

\begin{abstract}
The severe acute respiratory syndrome coronavirus-2 (SARS-CoV-2) causing the COVID-19 pandemic, has had an enormous effect on conventional clinical practice. Telemedicine has emerged as critical to the provision of healthcare services when reducing the transmission of COVID-19 among patients, families, and clinicians. It has been an essential tool for continuing care for patients with lower urinary tract symptoms (LUTS) during the COVID-19 pandemic and has been the link between socially distant patient contact. The aim of this perspective paper was to identify the strengths and limitations of technology-based care focusing on literature linked to patients with lower urinary tract symptoms (LUTS). We search PubMed and CINHAL Plus for grey literature and secondary research on LUTS and telemedicine during the COVID-19 pandemic. Publications dated between the year March 2020 and March 2021were searched. We gathered key specialist opinions in the field of LUTS from several countries around the world, including the countries that had been hit significantly with COVID-19. This perspective paper proposes that there is evidence to support the use of modern technology to facilitate continued healthcare services for patients with LUTS during the COVID-19 pandemic. Telemedicine has been recognised a crucial digital tool for diagnosis, treatment and follow-up appointments during a time of social distancing. Although there are many advantages of telemedicine, the older adult population and those economically disadvantaged with technology may not benefit from technology-based healthcare. The available literature on telemedicine during the COVID-19 pandemic has proven to be successful in the management of some patients with LUTS. It is certain that the COVID-19 pandemic has given telemedicine a significant drive for implementation now and in the immediate future. Robust data on long-term efficacy and safety of telemedicine is required to ensure there are governance protocols embedded when looking after patients with LUTS.
\end{abstract}

Keywords Telemedicine $\cdot$ LUTS $\cdot$ Urology $\cdot$ COVID-19

\section{Introduction}

The severe acute respiratory syndrome coronavirus-2 (SARS-CoV-2) causing the COVID-19 pandemic [1] disrupted societies all over the world. This has had an enormous

This article belongs to the Topical Collection: COVID-19 Health

Technology: Design, Regulation, Management, Assessment

Linda Collins

1.collins@sgul.kingston.ac.uk

Rajvinder Khasriya

rajvinder.khasriya@nhs.net

James Malone-Lee

james.malone-lee@ucl.ac.uk

1 Health, Social Care and Education, Kingston University and St George's University of London, London, UK

2 Department of Renal Medicine, Division of Medicine, University College London, London, UK effect on conventional clinical practice. Social distancing forced changes in the delivery of care in outpatient services so as to balance provision against while minimising risk of viral transmission to patients and health care professionals [2]. In these circumstances there is always a risk that what are perceived to be less pressing needs, such as the treatment of lower urinary tract symptoms (LUTS) and incontinence may be subordinated to more concerning problems such as cardiac disease with a COVID-19 diagnosis [3] or cancer treatment with adverse COVID-19 symptoms [4], as well as pre-existing respiratory diseases such as COPD in addition to COVID-19 [5]. This may affect patients and staff alike who will tend to weigh risk against need so that those with chronic diseases are not disadvantaged. Decisions on the distribution of resources will also tend to favour those sectors dealing with SARS-CoV-2 infection [6]. It is important that the clinicians working in the relevant specialities act to protect the interests of vulnerable patients who may be disadvantaged by these influences. One approach it to 
use technological advances to provide consultations that are safe. Real-time videoconferencing and telephone consultations, collectively termed telemedicine [7] are obvious options. Telemedicine is defined as a technology enabled care service providing care for patients with long term conditions that is convenient, accessible and cost-effective [8]. According to Calton et al. [9] telemedicine has emerged as critical to the provision of medical care when reducing the transmission of COVID-19 among patients, families, and clinicians. A digital patient is defined as a patient who uses and engages with mobile health technologies such as telemedicine [10]. Although there have been studies that have evaluated the effectiveness of telemedicine for service improvement [11-13], telemedicine is yet to be evaluated from the perspective of older adults with LUTS receiving care during the COVID-19 pandemic. The symptoms are debilitating but not life-threatening and may be triaged out of face-to-face provision. The aim of this evidence-based perspective was to identify the strengths and limitations of technology-based care and the future of telemedicine focusing on literature linked to patients with lower urinary tract symptoms (LUTS).

\section{Methodology}

We gathered key specialist opinions in the field of LUTS from several countries around the world, including the countries that had been hit significantly with COVID-19. The research questions, search procedure and inclusion and exclusion criteria are discussed below. This evidence-based perspective was guided by the following questions, what are the strengths and limitations of technology-based continuity of care for patients with lower urinary tract symptoms (LUTS)? What is the role of LUTS telemedicine in the future? What are the recommendations for quality assurance for long term use of telemedicine?

The evidence-based perspective was built using PubMed and CINHAL Plus databases. We decided to select all papers evaluating the use of telemedicine for patients diagnosed with LUTS. An electronic table (Table 1) was created by one of the authors to insert a list of the selected papers for outcome data, strengths, limitations and the future use of telemedicine for LUTS. The electronic table was double checked by the second author for accuracy, relevance and the risk of data bias using the ROBIS tool to assess risk of bias in systematic reviews [14]. Three authors reviewed the titles and abstracts of the records listed in each of the databases and selected the papers that were relevant to this evidencebased perspective. The papers were selected based upon their significance and were assessed in full text to review the findings.

\subsection{Inclusion and exclusion criteria}

The main search terms used in both databases were telemedicine, LUTS, Urology and COVID-19 and we searched for grey literature and secondary research dated between the year March 2020 and March 2021. The inclusion criteria included peer reviewed papers, with access to full text and related to telemedicine, LUTS, Urology and COVID19. Studies not linked to the inclusion criteria or focused on the related questions were excluded.

\section{Results}

Limited records were retrieved based upon the publication date and the nature of the topic. A total of five records were retrieved from PubMed and two from CINAHL Plus. The two papers retrieved from CINHAL Plus were duplicates found in the PubMed search and were removed. The remaining four papers were selected as they were relevant to "telemedicine", "LUTS", "Urology" and "COVID19" and provided data for our perspective. The process of literature retrieval and selection has been illustrated in a flow diagram (Fig. 1) which complied with the framework for reporting narrative synthesis [11]. Of the five papers included within this evidence-based perspective, four were review papers [15-18] and one was an observational study [19]. The authors who published the review papers evaluated existing literature on the effectiveness of telemedicine for patients with LUTs and indicated that telemedicine had been implemented successfully in several clinical scenarios during the pandemic. Out of the four review papers, one paper was an international narrative review of existing literature, with collaboration from leaders in the field of female and functional urology (FFU) from several countries around the world. Their goal was to develop a strategy that reorganises FFU activity, diagnosis and treatment during the COVID-19 pandemic based upon technology through the synthesis of evidence. The authors from the observational study examined their clinical activity and the use of telemedicine during a 7-week COVID-19 lockdown as part of departmental planning for continued urological services. Their findings highlighted the importance of flexible healthcare delivery with the use of telemedicine during the pandemic and in the future.

The COVID-19 pandemic has shown that telemedicine is a pertinent and accessible method for delivering efficient healthcare for patients and clinicians [20]. A recent study has shown a large proportion of patients are willing to keep a telemedical appointments scheduled during the COVID-19 pandemic [21]. The advantage of being 


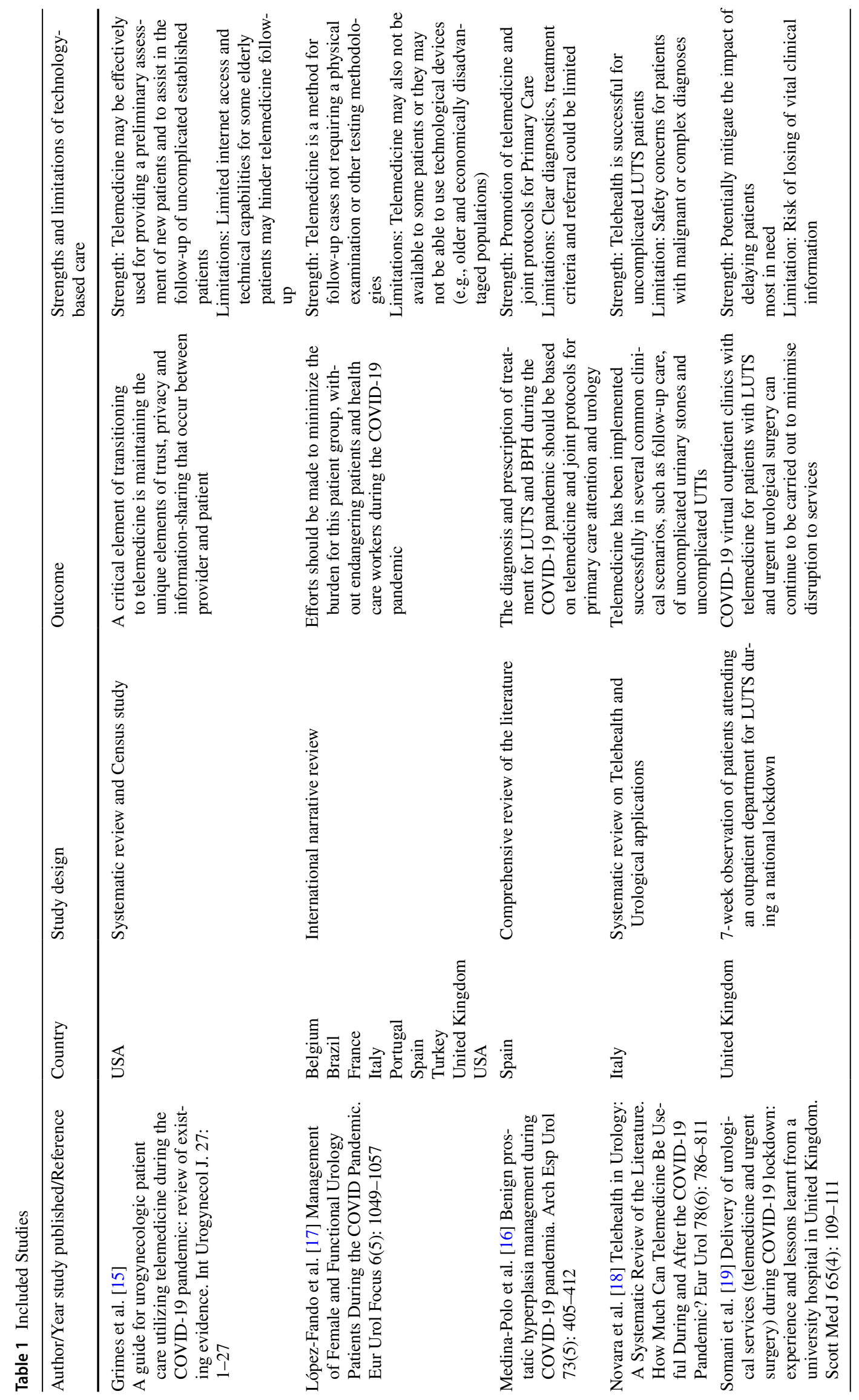


Fig. 1 Literature retrieval flow diagram

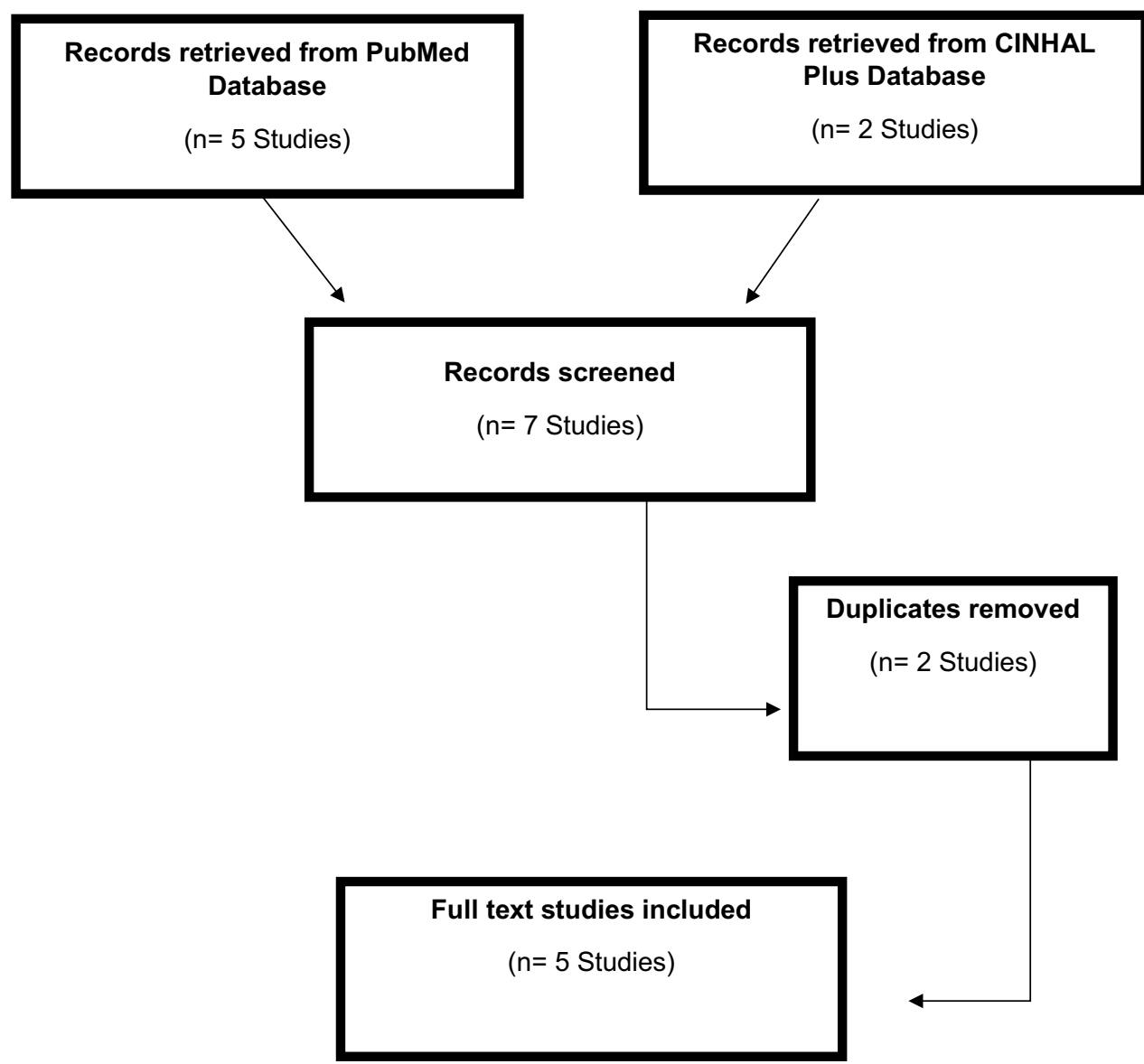

able to attend a follow-up appointment without attending a clinical unit has been recognised as an advancement in healthcare service delivery and mitigates the impact of delayed follow-up appointments for patients who require subsequent reviews [19]. López-Fando et al. [17] highlighted that telemedicine was an essential method for patient follow-up during the COVID-19 pandemic particularly for LUTS cases not requiring a physical examination or other testing methodologies. Medina-Polo et al. [16] also identified the advancement of telemedicine during the pandemic but were aware that telemedicine promoted the need for joint implemented policies for patients with LUTS in primary care. Although telemedicine has existed prior to COVID-19 in LUTS services, the recognition of it being an essential part of healthcare service delivery is merited. The LUTS clinic at Whittington Health North London, implemented telemedicine from the year 2010 , when the patient numbers grew beyond local tertiary referrals. It was evident then that LUTS and telemedicine was the way forward for managing patients nationally. Novara et al. [18] indicates that telehealth is successful for uncomplicated LUTS patients however, complex patients with chronic urinary tract infections (UTI) have been successfully managed by telemedicine supplemented by urine specimen examinations [22] which can be achieved at different times and close to the patients' locales. Somani et al. [19] have recognised through their observational study, that the COVID-19 pandemic has encouraged a greater openness to virtual outpatient services providing healthcare that minimises disruption to continuing care. Similarly, Grimes et al. [15] have laid emphasis on the multidimensional qualities of telemedicine, describing how the pandemic has forced a shift in how patients can receive continued, seamless care in more convenient and efficient way with high levels of patient satisfaction.

The publications have highlighted the fact that telemedicine may not be accessible to all, such as some in the older adult population, individuals who are economically disadvantaged, persons disadvantaged by language, cognition and technophobia although families and friends can help much with these $[15,17]$. Such persons are at risk of falling through the net, being lost to follow-up or harmed through information deficiencies [19]. This is particularly the case for patients with complex diagnoses [18] and LUTS hosts an increased representation of these groups. Some people buck the social trends and experience shyness and inhibitions when discussing personal matters across an electronic connection. With the advancements in health technologies 
and the move towards integrated healthcare management systems, there is a need to ensure that patients with LUTS are abreast of these technological changes. Healthcare professionals have a vital role when advocating the use of health technology, but more importantly have a duty of care to follow up with patients who lack digital literacy and adopt minimal use of digital apps and electronic devices for their healthcare management.

\section{Discussion}

\subsection{The future of LUTS telemedicine post COVID-19}

During the COVID-19 pandemic, Medina-Polo et al. [16] believed that the diagnosis and treatment for patients with LUTS should be based on telemedicine. Novara et al. [18] have identified that the future of telemedicine can be usefully implemented to guide patients in the decisionmaking process, diagnosis of LUTS and treatment selection for individual cases. Somani et al. [19] 7-week observational study highlighted that the use of telemedicine had risen steeply during the COVID-19 pandemic and is likely to become a sustainable reality post pandemic. The acceptance and integration of telemedicine in Urogynecology practice for patients with LUTS is possible, due to rapid advances in telecommunications and digital technology [15]. Patients with chronic UTI have relied on telemedicine and empirical antibiotic therapy as an effective method of treating the condition and lowering clinical pressure on health services [23]. It would seem that telemedicine will be playing a greater part healthcare service delivery as it is a practice that has currently expanded beyond traditional diagnostic and monitoring activities [18]. This is a good advancement, but López-Fando et al. [17] highlights the need to tread carefully into the world of telemedicine by working to minimise the special risks that will affect subsets of our patient population.

Telemedicine should continue to be used if it minimises disruption to services and patient care [19]. Transitioning to telemedicine for managing LUTS and pelvic floor disorders is critical for continuing conservative treatment [15]. Sacco et al. [24] acknowledge that virtual consultations providing clinical instructions and telemonitoring of patient symptoms is the way forward, but they lay emphasis on the importance of patient assessment, engagement and evaluation of telemedicine efficacy for long-term use. There is no doubt that telemedicine has contributed to efficient service provision, and in the case of patients with LUTS, has been an essential tool for patient contact when social distancing had been a major factor. Relying on face-to-face consultations as the main source of service provision may soon be recognised as past practice, as contemporary management of LUTS patients through digital technology is widely used. Somani, Pietropaolo et al. believe that clinicians and healthcare professionals should be trained on how to effectively use telemedicine prior to mass implementation, to maximise healthcare delivery but without compromising patient safety [19]. It is undeniable that healthcare professionals are relied upon to maintain patient safety and provide quality care. Telemedicine has a multifaceted approach, enabling care provision for patients with long term conditions that is convenient, accessible and economically sustainable [8]. As Somani et al. have mentioned, training is essential to maximise effective use of telemedicine, but the emphasis on patient safety outweighs mass implementation, and education, training and further professional development are significant factors for maximising the use of telemedicine.

\section{Recommendations for quality assurance for long term use of telemedicine}

There is published evidence that telemedicine has been implemented successfully in the management of uncomplicated LUTS [18]. The recommendations from Medina-Polo et al. literature review [16] suggests that the management of LUTS-Benign prostatic hyperplasia during and after the COVID-19 pandemic should consist of telemedicine and joint protocols for delivering telemedicine within Primary Care. It would be wise to ensure that proper feedback loops are established at this early stage so that our enthusiasm is not allowed to neglect unknown and unforeseen adverse consequences that nobody imagined. The findings from Novara et al. systematic review supports the concept of robust data collection on long-term efficacy, safety, and health economics, necessary for long term use of telemedicine for patients with LUTS [18]. Somani et al. recommend telemedicine for consultations as implemented within their observational study, recognising it as a useful method to prioritise the patients most in need of urgent care and to maximise a flexible healthcare service delivery [19]. Further understanding of LUTS and telemedicine is continually being uncovered, and patient experience of LUTS and telemedicine during the COVID19 pandemic is imminent. With new evidence emerging, policy and guidelines on LUTS and telemedicine is needed to ensure that equitable digital care is accessible to all through the national promoted technology enabled care services [8]. Further research is needed to quantify the impact of LUTS telemedicine in comparison to face-to-face consultations and the advantages and disadvantages of its long-term use. 


\section{Conclusion}

We do not know whether COVID-19 will be present for the years to come. Nevertheless, we should be prepared to provide efficient healthcare service throughout. The available literature on telemedicine during the COVID-19 pandemic has provided encouragement for the future use of these methods for the care of patients with LUTS. It would see that some complex bladder conditions are suitable for telehealth. We are optimistic about the future potential but emphasise the importance of building in thorough safety surveillance feedback systems that ensure quality assurance for clinicians and health care professionals as well as patients receiving telehealth services.

Authors' contributions Collins: Data search, data review, manuscript writing/editing. Khasriya: Data review, manuscript writing/editing. Malone-Lee: Data review, manuscript writing/editing.

Funding There were no funding or incentives for conducting this perspective paper.

Availability of data and material The secondary data used and/or analysed for this perspective paper are available and accessible from the PubMed and CINAHL Plus.

\section{Declarations}

Conflict of interest There were no competing interests.

Open Access This article is licensed under a Creative Commons Attribution 4.0 International License, which permits use, sharing, adaptation, distribution and reproduction in any medium or format, as long as you give appropriate credit to the original author(s) and the source, provide a link to the Creative Commons licence, and indicate if changes were made. The images or other third party material in this article are included in the article's Creative Commons licence, unless indicated otherwise in a credit line to the material. If material is not included in the article's Creative Commons licence and your intended use is not permitted by statutory regulation or exceeds the permitted use, you will need to obtain permission directly from the copyright holder. To view a copy of this licence, visit http://creativecommons.org/licenses/by/4.0/.

\section{References}

1. Chen N, et al. Epidemiological and clinical characteristics of 99 cases of 2019 novel coronavirus pneumonia in Wuhan, China: a descriptive study. Lancet. 2020;395(10223):507-13.

2. Figueiredo AA, et al. Interim Guidance for Urodynamic Practice during COVID-19 Pandemic, in Int Braz J Urol. 2021:178-84.

3. Kovach CP, Perman SM. Impact of the COVID-19 pandemic on cardiac arrest systems of care. Curr Opin Crit Care. 2021;27(3):239-45.

4. Bakouny Z, et al. COVID-19 and cancer: current challenges and perspectives. Cancer Cell. 2020;38(5):629-46.
5. Turan $\mathrm{O}$, et al. Clinical characteristics and outcomes of hospitalized COVID-19 patients with COPD. Expert Rev Respir Med. 2021:1-8.

6. Hillery S. Taking care of continence patients during the COVID19 pandemic. Br J Nurs. 2020;29(22):1318-24.

7. Buvik A, et al. Cost-effectiveness of telemedicine in remote orthopedic consultations: randomized controlled trial. J Med Internet Res. 2019;21(2): e11330.

8. NHS. Technology Enabled Care Services (TECS). 2021 [cited 2021 March 3 2021]; Available from: https://www.england.nhs. uk/tecs/.

9. Calton B, Abedini N, Fratkin M. Telemedicine in the time of coronavirus. J Pain Symptom Manage. 2020;60(1):e12-4.

10. Kalhori SR, Bahaadinbeigy K, Deldar K, Gholamzadeh M, Hajesmaeel-Gohari S, Ayyoubzadeh SM. Digital health solutions to control the COVID-19 pandemic in countries with high disease prevalence: Literature review. J Med Internet Res. 2021;23(3):e19473.

11. Jarvis-Selinger $\mathrm{S}$, et al. Clinical telehealth across the disciplines: lessons learned. Telemed J E Health. 2008;14(7):720-5.

12. Steel K, Cox D, Garry H. Therapeutic videoconferencing interventions for the treatment of long-term conditions. J Telemed Telecare. 2011;17(3):109-17.

13. Bashshur RL, et al. Sustaining and realizing the promise of telemedicine. Telemed J E Health. 2013;19(5):339-45.

14. Whiting $\mathrm{P}$, et al. ROBIS: A new tool to assess risk of bias in systematic reviews was developed. J Clin Epidemiol. 2016;69:225-34.

15. Grimes CL, et al. A guide for urogynecologic patient care utilizing telemedicine during the COVID-19 pandemic: review of existing evidence. Int Urogynecol J. 2020;31(6):1063-89.

16. Medina-Polo J, et al. [Benign prostatic hyperplasia management during COVID-19 pandemia.]. Arch Esp Urol. 2020;73(5):405-12.

17. López-Fando L, et al. Management of female and functional urology patients during the COVID pandemic. Eur Urol Focus. 2020;6(5):1049-57.

18. Novara G, et al. Telehealth in urology: a systematic review of the literature. How much can telemedicine be useful during and after the COVID-19 pandemic? Eur Urol. 2020;78(6):786-811.

19. Somani BK, et al. Delivery of urological services (telemedicine and urgent surgery) during COVID-19 lockdown: experience and lessons learnt from a university hospital in United Kingdom. Scott Med J. 2020;65(4):109-11.

20. Gadzinski AJ, et al. Implementing telemedicine in response to the COVID-19 pandemic, in J Urol. 2020:14-6.

21. Boehm K, et al. Telemedicine online visits in urology during the COVID-19 pandemic-potential, risk factors, and patients' perspective. Eur Urol. 2020;78(1):16-20.

22. Sathiananthamoorthy $\mathrm{S}$, et al. Reassessment of routine midstream culture in diagnosis of urinary tract infection. J Clin Microbiol. 2019;57(3).

23. Mehrotra A, et al. A comparison of care at e-visits and physician office visits for sinusitis and urinary tract infection. JAMA Intern Med. 2013;173(1):72-4.

24. Sacco E, et al. Extensive impact of COVID-19 pandemic on pelvic floor dysfunctions care: A nationwide interdisciplinary survey. Neurourol Urodyn. 2021;40(2):695-704.

Publisher's Note Springer Nature remains neutral with regard to jurisdictional claims in published maps and institutional affiliations. 